pometric measurements of the fetus and neonate', come to the conclusion that there is no clear indication that the prior use of oral contraceptives alters the risk for chromosomally abnormal offspring: a finding contrary to that of some earlier studies.

Chapter 6, 'Structural rearrangements of the chromosomes in man', is a valuable appreciation of structural rearrangements with respect to reproductive fitness and pregnancy outcome. Chapter 7, 'Euploid structural rearrangements in the mentally retarded', is important in highlighting the fact that there is a six- to sevenfold increase in reciprocal translocations and nearly a fourteenfold increase in inversion in the mentally retarded studied in this survey (1037 individuals). It seems probable that de novo structural rearrangements account for much of this increase.

Chapter 9 is concerned particularly with the findings in a subsample of $\mathbf{4 0 0}$ children from the study described in Chapter 8; with special emphasis to definition of 'polymorphism' and racial difference. There are marked differences in the frequency of some polymorphisms between White and Black children. Chapter 10 is also concerned with polymorphisms.

Chapter 11 is concerned with the 'Heritability of chromosome banding variants'. Chapter 12, 'Population cytogenetics of leukemia', is an authoritative and clearly written review of the present state of know ledge regarding chromosome abnormality in leukaemia. The use of optical methods for detecting DNA synthesis and sister chromatid exchange is discussed in Chapter 13. Sister chromatid exchanges are becoming increasingly used as indices of chromosomal damage and repair and characterise a number of human diseases. The method outlined in this chapter is a very sensitive indicator of sister chromatid exchange.

In Chapter 14, 'The nonrandomness of human chromosome abnormalities', there are some interesting and provocative comments on the epidemiology of human chromosome abnormality. 'Chromosome registers-problems and perspectives', Chapter 15, is a penetrating review which could apply to disease registers in general. The author's attitude seems indicated by his quote of J. M. Weddel: 'The critical question is: can this be done in any other way? If the answer is yes, then it is probable that a register is a luxury'. Chapter 16, 'Amniocentesis and selective abortion for trisomy 21 in the light of the natural history of pregnancy and fetal survival', gives an interesting account of the natural history of chromosome abnormality from conception to postnatal existence, with special reference to trisomy 21 , and is particularly pertinent to the use of fetal chromosome analysis as a screening procedure. The epidemiology of Down's syndrome is further discussed in Chapter 17 , 'Cluster analysis and racial differences in risk of Down's syndrome', Chapter 18, 'Maternal radiation and trisomy 21' and Chapter 19, 'Parental factors in Down's syndrome-results of the second Baltimore case - control study'. Much of the work outlined in Chapters 17 to 19 is discussed again in the final chapter entitled 'Human population cytogenetics: comments on racial differences in frequency of $Q$ chromosome abnormalities, putative clustering in $\mathrm{w}$ Down's syndrome, and radiation studies'. These $\overrightarrow{0}$ authors, the editors of this volume, come to the conclusion, 'that there is still no definitive evidence in $\vec{\omega}$ the literature for consistent differences between races in the prevalence at birth of Down's syndrome at any maternal age quinquennia'. They also say 'the $\vec{v}$ trend to date suggests a lower rate of XYY's in black newborns and of XXY males in white newborns, but $\infty$ clearly further data are needed'. They are not convinced that there is reason to believe that there is 'Variation in incidence in Down's syndrome in space and/or time'. They do, however, view published data on radiation as highly suggestive, but not conclusive that radiation experience (diagnostic and/or therapeutic) may increase the frequency of chromosome abnormality consequent upon meiotic nondisjunction.

Although a useful source of reference this volume contains little work not published elsewhere, often in a more readable form. At $£ 12.40$ it is expensive.

C. E. Blank

Dermatoglyphics in Medical Disorders.

By Blanka Schaumann and Milton Alter. (Pp. xi $+\widehat{\varrho}$ 258; 74 figures + 51 tables. Cloth DM54,90; US 후 \$22.50.) Berlin, Heidelberg, New York: SpringerVerlag. 1976.

The value of dermatoglyphs as an aid to diagnosis 응 increases pari passu with the discovery of new $\rightarrow$ conditions associated with unusual configurations or pattern frequencies. During recent years $9 p+$ trisomy $N$ and the syndrome of Coffin, Siris, and Wegienka $O$ have been added to their number. Those able to $N$ analyse prints however, are few and far between. $\underset{\mathrm{C}}{\mathrm{N}}$ Prints can, of course, be sent for analysis but even the $O$ ability to take prints sufficiently well for them to be readable and sufficiently complete (containing not only $\frac{0}{\Phi}$ all patterns but all associated triradii) is rare. This is $\stackrel{?}{\rightarrow}$ a pity because, though a diagnosis can seldom be 0 based on prints alone, they are useful aids to diag- $\bar{O}$ nosis. 
In this book the genetics and anthropological aspects of dermatoglyphs are not discussed. Topological classification and analysis is explained and described briefly, and the value and construction of dermatoglyphic dictionaries is not described, nor is there any mention of discriminant methods in diagnosis. Such items are beyond the scope of the authors' intention which is to provide an 'illustrated guide' and explanation of the methods of taking prints and of identifying and recording them, and also, an account of the dermatoglyphic findings in those conditions in which deviations from the normal range are established and are of some diagnostic value. The book is lucidly written and well illustrated. About a third of the book is devoted to an account of diseases, mainly those with chromosome disorders and limb anomalies, characterised by abnormal dermatoglyphic findings. There are Tables of pattern and ridge count frequencies in the normal population and in the various conditions. In a book of this kind, adequate illustrations and Tables are essential and these are well chosen and adequately supplied.

There are a few minor errors. One important error is the quotation from Dieker and Opitz $(1969$, p. 76), who stated that the topological formula $(T+1=$ $L+D)$ does not apply in zygodactyly because 'variable degrees of severity of this defect may reduce the number of triradii shared by the two affected digits to zero'. It does, of course, apply, and Penrose was at great pains to point out in some detail how it applied in zygodactyly.

The book can be recommended to doctors and others who, it is hoped, will wish to make practical use of dermatoglyphs as an aid to diagnosis. It is well produced, adequately supplied with references, but, like most books published abroad nowadays, expensive for British readers.

B. W. RICHARDS

\section{Medico-Social Management of Inherited Metabolic Disease.}

Edited by D. N. Raine. (Pp. $x+300$; Figures + Tables. £9.95.) Lancaster: MTP Press. 1977.

This monograph records the proceedings of the 13th Symposium of the Society for the Study of Inborn Errors of Metabolism edited and enlarged by $\mathrm{Dr}$ D. N. Raine. In the past it has sometimes been difficult accurately to evaluate the importance of screening for inherited metabolic diseases, considering the rarity of individual disorders and the considerable expense of setting up the laboratory and administrative services necessary to involve whole populations. This volume will go far to put into perspective the present situation and future prospects.

Biochemical disorders are inherently attractive since they promise the systematic uncovering of logical and predictable metabolic pathways linking genes with clinical phenomena. This approach is much appreciated by geneticists and clinicians who see in it the best way to identify homozygotes and heterozygotes and to avoid the confusion of genetic heterogeneity. Furthermore, the urge to reduce the frequency of such disorders in future children is a natural and humane consequence of treating children with metabolic disorders and supporting their families. Increasingly, prenatal diagnosis allows conceptions after that of the proband to be monitored and, in the case of an affected fetus, selective termination of pregnancy may be carried out. However, in the majority of cases there is no family history to alert the obstetrician of the need for prenatal diagnosis and even if there is, a lack of suitable biochemical techniques as in phenylketonuria and cystic fibrosis prevents this approach. The current phase of rapid technological advance means that many more disorders will be able to be diagnosed prenatally and it will become important to be able to detect carriers (after adequate education and counselling) so as to be able to offer prenatal diagnosis for first affected fetuses. The early detection of inborn errors of metabolism in infancy has an important role even when they are untreatable since genetic counselling will allow couples to avoid the conception of further affected children or, when appropriate, seek prenatal diagnosis. However, one must be suspicious of organised programmes to detect the untreatable.

These and other problems are competently discussed in this monograph in sensibly defined sections by leaders in the field. 'The nature and size of the problem' precedes the limitations of 'The present methods of management' while the 'Community reaction to present practice' is discussed against a background of parental involvement and the role of genetic counselling clinics and 'eugenic abortion'. The editor had laid particular stress on 'Aspects of management requiring central policy' since largescale screening requires very careful administration and adequate funding. The volume contains as a bonus the fourth Milner lecture by Dr T. A. Perry entitled 'The biochemical autopsy: a tool for studies of genetically determined brain disorders'.

This volume will be of considerable interest to everyone concerned with human biochemical disorders but will be particularly important for those who have responsibility for planning and carrying out screening programmes.

RODNEY HARRIS 